\title{
Variable Gain Takagi-Sugeno Fuzzy Logic Controllers
}

\author{
Reghunadhan RAJESH, M. Ramachandra KAIMAL \\ Dept. of Computer Science and Engg., Bharathiar University \\ Coimbatore 641046, India \\ Dept. of Computer Science, University of Kerala \\ Thiruvananthapuram 695581, India \\ e-mail: kollamrajeshr@ieee.org
}

Received: November 2005

\begin{abstract}
Most of the Takagi-Sugeno Fuzzy (TSF) systems found in the literature have only used linear functions of input variables as rule consequent and can be called as TSF Models with Fixed Coefficient (TSFMFC). This paper presents TSF model with variable coefficient (TSFMVC) which can more closely approximate a class of nonlinear systems, nonlinear dynamic systems, and nonlinear control systems. It is also shown that TSFMFC is a special case of TSFMVC. Moreover Variable Gain TSF Controller (VGTSFC) is defined and it performs better, as shown by the simulation results, when compared with Fixed Gain TSF Controller (FGTSFC).
\end{abstract}

Key words: fuzzy controller, Takagi-Sugeno fuzzy models with fixed coefficient, Takagi-Sugeno fuzzy models with variable coefficient, fixed gain Takagi-Sugeno fuzzy controller, variable gain Takagi-Sugeno fuzzy controller.

\section{Introduction and Problem Statement}

Takagi-Sugeno fuzzy systems have widely been employed in the fields of control and modeling. In the context of control, the question is whether a fuzzy controller can be constructed to approximate any desired control solution with enough accuracy, whereas, in the context of modeling the issue is whether a fuzzy model can be established which is capable of approximating any physical system as accurately as one desires. Model-Based control design assumes the existence of a mathematical model of the plant. The controller is designed to modify the behavior of the plant and achieve some desired performance. To this end, a systematic way to construct a model, mapping the inputs to the outputs is needed.

Most of the Takagi-Sugeno fuzzy systems found in the literature (Joh et al., 1998; Li et al., 2003; Takagi and Sugeno, 1985; Tanaka and Sugeno, 1992; Wang et al., 1996; Wang et al., 2003; Wang et al., 1995; Ying, 1998) have only used linear functions of input variables as rule consequent (i.e., linear rule consequent) and can be called as Takagi-Sugeno Fuzzy Models with Fixed Coefficient (TSFMFC). It simply means that the coefficients of state variables in the consequents of each rule are fixed constants. This 
paper presents an extended Takagi-Sugeno fuzzy model named as Takagi-Sugeno fuzzy model with variable coefficient (TSFMVC) and it is proved that it can approximate a class of nonlinear systems, nonlinear dynamic systems, and nonlinear control systems. It is also shown that TSFMFC is a special case of TSFMVC.

Takagi-Sugeno fuzzy controllers designed using parallel distributed technique found in the literature (Wang et al., 1995) uses linear functions of input variables as rule consequents and may be called as Fixed Gain Takagi-Sugeno Fuzzy Controller (FGTSFC). This paper also presents an extended T-S fuzzy controller called as Variable Gain Takagi-Sugeno Fuzzy Controller (VGTSFC) using Parallel distributed Compensation. A nonlinear benchmark problem corresponding to the physical system that has been built by Dr. Bernstein and his co-workers (Bernstein, 1998; Bupp et al., 1995) at the University of Michigan namely the Translational Oscillation by a Rotational Actuator (TORA) (A1leyne, 1998; Jankovic et al., 1996; Li et al., 2000; Kanellakopoulos and Zhao, 1995; Margaliot and Langholz, 2004; Rand et al., 1992) is simulated and the results show that VGTSFC performs better than FGTSFC.

This paper is organized as follows. Section 2 deals with the review of T-S fuzzy models with fixed coefficients and the corresponding fixed gain T-S fuzzy controller. Section 3 presents the proposed Takagi-Sugeno Fuzzy Models with Variable Coefficient (TSFMVC). Section 4 deals with the analysis of approximation of a class of nonlinear systems using TSFMVC. Section 5 deals with the proposed VGTSFC, its approximation and comparison with FGTSFC. In Section 6 TORA system is simulated using FGTSFC, VGTSFC, and linear controller (linearized around the origin) and the results are compared.

\section{Review of T-S Fuzzy Models with Fixed Coefficients (TSFMFC) and the Corresponding Fixed Gain T-S Fuzzy Controller (FGTSFC)}

A general representation of T-S model (Wang et al., 2003) with fixed coefficient (TSFMFC) is presented in this section.

Suppose that the nonlinear function $f(x): R^{n} \rightarrow R$ is defined over a compact region $D \subset R^{n}$ with the following assumptions:

$A_{1}: f(0)=0$

$A_{2}: f \in C_{1}^{2}$. Therefore, $f, \frac{\partial f}{\partial x}$ and $\frac{\partial^{2} f}{\partial x^{2}}$ are continuous and therefore bounded over $D$.

$A_{3}: f(x)$ can approximated in the form of $f_{j_{1} j_{2} \ldots j_{n}}(x)=a_{j_{1} j_{2} \ldots j_{n}} x$ in each local region $D_{j_{1} j_{2} \ldots j_{n}}=\left\{x \mid x \in D, j_{i} \epsilon \leqslant x_{i} \leqslant\left(j_{i}+1\right) \epsilon \forall i\right\}$, where $j_{i}$ are integers and $\epsilon$ is a small chosen positive number.

Then $f(x)$ can be expressed by linear Takagi-Sugeno fuzzy systems. The main feature of linear Takagi-Sugeno fuzzy systems is to express the local properties of each fuzzy implication (rule) by a linear function. The overall fuzzy system is achieved by fuzzy "blending" of these linear functions.

Then the linear Takagi-Sugeno fuzzy rules are as follows:

$$
R_{j_{1} j_{2} \ldots j_{n}}: \text { IF } x_{1} \text { is about } j_{1} \epsilon \ldots \text { and } x_{n} \text { is about } j_{n} \epsilon \text { THEN } \hat{f}=a_{j_{1} j_{2} \ldots j_{n}} x \text {, }
$$


where $x=\left[x_{1}, x_{2}, \ldots, x_{n}\right]^{T}$ are the function variables and $\hat{f}=a_{j_{1} j_{2} \ldots j_{n}} x$ is the consequence of the $j_{1} j_{2} \ldots j_{n}{ }^{\text {th }}$ IF-THEN rule. [It should be noted that, " $x_{i}$ is about $j_{i} \epsilon$ " is same as telling that " $x_{i}$ takes the membership function $M_{j_{i}}\left(x_{i}\right)$. "]

The possibility of firing for the $j_{1} j_{2} \ldots j_{n}{ }^{\text {th }}$ rule is given by the product of all membership functions associated with the $j_{1} j_{2} \ldots j_{n}{ }^{\text {th }}$ rule.

$$
h_{j_{1} j_{2} \ldots j_{n}}(x)=\prod_{i=1}^{n} M_{j_{i}}\left(x_{i}\right)
$$

where the membership function for $x_{i}$ is given as

$$
M_{j_{i}}\left(x_{i}\right)= \begin{cases}1-\frac{\left|x_{i}-j_{i} \epsilon\right|}{\epsilon}, & \left|x_{i}-j_{i} \epsilon\right|<\epsilon \\ 0, & \text { else where. }\end{cases}
$$

It is noted that $h_{j_{1} j_{2} \ldots j_{n}}(x)$ have already been normalized, i.e., $h_{j_{1} j_{2} \ldots j_{n}}(x) \geqslant 0$ and $\sum_{j_{1} j_{2} \ldots j_{n}} h_{j_{1} j_{2} \ldots j_{n}}(x)=1$.

Then by using center of gravity method for defuzzification, we can represent the T-S model as

$$
f(x)=\sum_{j_{1} j_{2} \ldots j_{n}} h_{j_{1} j_{2} \ldots j_{n}}(x) a_{j_{1} j_{2} \ldots j_{n}} x .
$$

Theorem 1 (Wang et al., 2003). For a smooth nonlinear function $f(x): R^{n} \rightarrow R$ satisfying Assumptions $A_{1}, A_{2}$ and $A_{3}$, defined on a compact region, it can be approximated, to any degree of accuracy, by a linear T-S fuzzy system.

\subsection{TSFMFC for Nonlinear Dynamic Systems}

The Takagi-Sugeno fuzzy model with fixed coefficient (TSFMFC) is used to describe dynamic systems. It is of the following form:

$$
R_{i} \text { : IF } x_{1}(t) \text { is } M_{1 i} \ldots \text { and } x_{n}(t) \text { is } M_{n i} \text { THEN } \dot{x}(t)=A_{i} x(t),
$$

where $x^{T}(t)=\left[x_{1}(t), x_{2}(t), \ldots, x_{n}(t)\right]$ are the system states. $i=1,2, \ldots, r$ and $r$ is the number of IF-THEN rules. $M_{i j}$ are fuzzy sets and $\dot{x}(t)=A_{i} x(t)$ are the consequence of the $i$-th IF-THEN rule.

By using center of gravity method for defuzzification, the T-S model can be represented as

$$
\dot{x}=\hat{f}(x)=\sum_{i=1}^{r} h_{i}(x) A_{i} x,
$$

where $h_{i}(x)$ is the possibility for the $i$-th rule to fire.

Consider the nonlinear system:

$$
\dot{x}=f(x),
$$


where $f(x)$ is a vector field defined over compact region $D \subset R^{n}$ with the following assumptions:

$B_{1}: f(0)=0$, i.e., the origin is an equilibrium point.

$B_{2}: f \in C_{n}^{2}$. Therefore, $f, \frac{\partial f}{\partial x}, \frac{\partial^{2} f}{\partial x^{2}}$ are continuous and bounded over $D$.

COROLLARY 1 (Wang et al., 2003). For any smooth nonlinear system (5) satisfying the assumptions $B_{1}$ and $B_{2}$, it can be approximated, to any degree of accuracy, by a T-S model (4).

Similarly, smooth nonlinear control system $\dot{x}=f(x)+g(x) u$ can also be approximated using a T-S fuzzy model $\dot{x}=\sum_{i=1}^{r} h_{i}(x)\left(A_{i} x+B_{i} u\right)$. By treating u as extraneous system state, we can also approximate the smooth nonlinear control system $\dot{x}=f(x, u)$ by T-S fuzzy model $\dot{x}=\sum_{i=1}^{r} \hat{h}_{i}(x, u)\left(A_{i} x+B_{i} u\right)$. In this case, the fuzzy rule is of the following form:

$$
\begin{gathered}
\text { Rule } i: \quad \text { IF } x_{1}(t) \text { is } M_{i 1}, \ldots, \text { and, } x_{n}(t) \text { is } M_{i n}, \\
u_{1}(t) \text { is } N_{i 1}, \ldots \text {, and } u_{m}(t) i s N_{i m} \\
\text { THEN } \dot{x}=A_{i} x(t)+B_{i} u(t)
\end{gathered}
$$

where $x^{T}(t)=\left[x_{1}(t), x_{2}(t), \ldots, x_{n}(t)\right]$ are the system states and $u^{T}(t)=\left[u_{1}(t), u_{2}(t)\right.$, $\left.\ldots, u_{m}(t)\right]$ are system inputs. $i=1,2, \ldots, r$ and $r$ is the number of IF-THEN rules. $M_{i j}$, $N_{i j}$ are fuzzy sets and $\dot{x}(t)=A_{i} x(t)+B_{i} u(t)$ is the consequence of the $i$-th IF-THEN rule. $\hat{h}_{i}(x, u)=\prod_{j=1}^{n} M_{i j}\left(x_{i}(t)\right) \prod_{k=1}^{m} N_{i k}\left(u_{k}(t)\right)$ is the possibility for the $i$-th rule to fire.

\subsection{FGTSFC Using PDC Technique}

The structure of FGTSFC using parallel distributed compensation (PDC) (Wang et al., 1996; Wang et al., 1995) consists of the fuzzy rules of the given below:

Rule $i$ : IF $x_{1}(t)$ is $M_{i 1}, \ldots, x_{n}(t)$ is $M_{i n}$, THEN $u(t)=K_{i} x(t)$,

where $i=1,2, \ldots, s$. The output of the PDC controller is

$$
u=\sum_{j=1}^{r} h_{j}(x) K_{j} x .
$$

Theorem 2 (Wang et al., 2003). Any smooth nonlinear state feedback controller $u=$ $K(x)$ where $x$ is defined over a compact region can be approximated, to any degree of accuracy, by a PDC controller (7). 


\section{T-S Fuzzy Models with Variable Coefficient (TSFMVC)}

A general representation of TSFMVC is as described below. Suppose that the nonlinear function $f(x): R^{n} \Rightarrow R$ is defined over a compact region $D \subset R^{n}$ with the following assumptions:

$P_{1}: f(0)=0$

$P_{2}: f \in C_{1}^{2}$. Therefore, $f, \frac{\partial f}{\partial x}$ and $\frac{\partial^{2} f}{\partial x^{2}}$ are continuous and therefore bounded over D.

$P_{3}: f(x)$ can be approximated as $f_{j_{1} j_{2} \ldots j_{n}}(x)=a_{j_{1} j_{2} \ldots j_{n}}(x) x$ in the region $D_{j_{1} j_{2} \ldots j_{n}}$ $=\left\{x / x \in D, j_{i} \epsilon \leqslant x_{i} \leqslant\left(j_{i}+1\right) \epsilon \forall i\right\}$, where $a(x), a_{j_{1} j_{2} \ldots j_{n}}(x)$ are continuous, $x=\left[x_{1}, x_{2}, \ldots, x_{n}\right]^{T}, j_{i}$ are integers, and $\epsilon$ is a small positive number.

Then $f(x)$ can be approximated by Takagi-Sugeno Fuzzy Model with Variable Coefficient (TSFMVC). The rules of TSFMVC is of the form:

$$
\begin{aligned}
\text { Rule } j_{1} j_{2} \ldots j_{n}: & \text { IF } x_{1} \text { is about } j_{1} \epsilon \ldots \text { and } x_{n} \text { is about } j_{n} \epsilon \\
& \text { THEN } \hat{f}(x)=a_{j_{1} j_{2} \ldots j_{n}}(x) x
\end{aligned}
$$

The possibility of firing for the $j_{1} j_{2} \ldots j_{n}{ }^{\text {th }}$ rule is given by the product of all membership functions associated with the $j_{1} j_{2} \ldots j_{n}{ }^{\text {th }}$ rule and is $h_{j_{1} j_{2} \ldots j_{n}}(x(t))=$ $\prod_{i=1}^{n} M_{j_{i}}\left(x_{i}(t)\right)$ where the membership function for $x_{i}$ is given by (8). It is assumed that $h_{j_{1} j_{2} \ldots j_{n}}(x)$ have already been normalized, i.e., $h_{j_{1} j_{2} \ldots j_{n}}(x) \geqslant 0$ and $\sum_{j_{1} j_{2} \ldots j_{n}} h_{j_{1} j_{2} \ldots j_{n}}(x)=1$.

$$
M_{j_{i}}\left(x_{i}\right)= \begin{cases}1-\frac{\left|x_{i}-j_{i} \epsilon\right|}{\epsilon}, & \left|x_{i}-j_{i} \epsilon\right|<\epsilon \\ 0, & \text { else where }\end{cases}
$$

Then by using center of gravity method for defuzzification, the TSFMVC can be represented as:

$$
y=\hat{f}(x)=\sum_{j_{1} j_{2} \ldots j_{n}} h_{j_{1} j_{2} \ldots j_{n}}(x) a_{j_{1} j_{2} \ldots j_{n}}(x) x
$$

\section{Analysis of Approximation}

\subsection{Approximation of a Function by T-S Fuzzy Model with Variable Coefficient (TSFMVC)}

In this section, it is proved the fact that any smooth nonlinear function satisfying the assumptions $P_{1}, P_{2}$, and $P_{3}$ can be approximated, to any degree of accuracy, using the TSFMVC. In the following discussions, only one of such regions $D_{j_{1} j_{2} \ldots j_{n}}$ is concentrated by assuming that $x \in D_{j_{1} j_{2} \ldots j_{n}}$. In the following, for simplicity, $\phi$ is substituted instead of $j_{1} j_{2} \ldots j_{n}$. 
Consider $e(x)$, the approximation error between $f(x)$ and $\hat{f}(x)$

$$
\begin{aligned}
\|e(x)\| & =\left\|f(x)-\sum_{\phi} h_{\phi}(x) a_{\phi}(x) x\right\| \\
& =\left\|f(x)-\sum_{\phi} h_{\phi}(x) a_{\phi}\left(x_{\phi}\right) x_{\phi}+\sum_{\phi} h_{\phi}(x)\left(a_{\phi}\left(x_{\phi}\right) x_{\phi}-a_{\phi}(x) x\right)\right\| \\
& \leqslant\left\|f(x)-\sum_{\phi} h_{\phi}(x) f\left(x_{\phi}\right)\right\|+\left\|\sum_{\phi} h_{\phi}(x)\left(a_{\phi}\left(x_{\phi}\right) x_{\phi}-a_{\phi}(x) x\right)\right\| \\
& \leqslant \sum_{\phi} h_{\phi}(x)\left\|f(x)-f\left(x_{\phi}\right)\right\|+\sum_{\phi} h_{\phi}(x)\left\|\left(a_{\phi}\left(x_{\phi}\right) x_{\phi}-a_{\phi}(x) x\right)\right\| \\
& \leqslant \max _{\phi}\left\|f(x)-f\left(x_{\phi}\right)\right\|+\max _{\phi}\left\|\left(a_{\phi}\left(x_{\phi}\right) x_{\phi}-a_{\phi}(x) x\right)\right\| .
\end{aligned}
$$

Since $x \in D_{j_{1} j_{2} \ldots j_{n}}$, the distance between $x$ and any vertex point of $D_{j_{1} j_{2} \ldots j_{n}}$ is less than $\sqrt{n} \epsilon$, i.e., $\left|x-x_{j_{1} j_{2} \ldots j_{n}}\right| \leqslant \sqrt{n} \epsilon$, and since $f(x)$ and $a(x)$ are continuous over $x$, it is possible to make $e(x)$ arbitrarily small by just reducing $\epsilon$.

Therefore, the following theorem is obtained by summarizing the results above.

Theorem 3. For a smooth nonlinear function $f(x): R^{n} \rightarrow R$ satisfying assumptions $P_{1}$, $P_{2}$, and $P_{3}$ can be approximated, to any degree of accuracy, by a T-S Fuzzy Model with Variable Coefficient (TSFMVC).

\subsection{Approximation of Nonlinear Dynamic Systems by TSFMVC}

TSFMVC in the following form can be used to describe dynamic systems.

Rule $j_{1} j_{2} \ldots j_{n}: \quad$ IF $x_{1}$ is about $j_{1} \epsilon \ldots$ and $x_{n}$ is about $j_{n} \epsilon$

$$
\text { THEN } \dot{x}=A_{j_{1} j_{2} \ldots j_{n}}(x) x,
$$

where $x=\left[x_{1}, x_{2}, \ldots, x_{n}\right]^{T}$ are the function variables and $\dot{x}=A_{j_{1} j_{2} \ldots j_{n}}(x) x$ is the consequence of the $j_{1} j_{2} \ldots j_{n}{ }^{\text {th }}$ IF-THEN rule.

By using weighted average defuzzification, the TSFMVC can be represented as (10), where $h_{i}(x)$ is the possibility for the $i^{t h}$ rule to fire.

$$
\dot{x}=\hat{f}(x)=\sum_{j_{1} j_{2} \ldots j_{n}} h_{j_{1} j_{2} \ldots j_{n}}(x) A_{j_{1} j_{2} \ldots j_{n}}(x) x .
$$

Consider the nonlinear system:

$$
\dot{x}=f(x)
$$

where $f(x)$ is a vector field defined over compact region $D \subset \Re^{n}$ with the following assumptions:

$S_{1}: f(0)=0$, i.e., the origin is an equilibrium point. 
$S_{2}: f \in C_{n}^{2}$. Therefore, $f, \frac{\partial f}{\partial x}$ and $\frac{\partial^{2} f}{\partial x^{2}}$ are continuous and therefore bounded over $D$.

Suppose $f(x)$ can be written as $\left[f_{1}(x) \ldots f_{n}(x)\right]^{T}$. What is meant by approximation is finding a T-S fuzzy model $\hat{f}(x)=\left[\hat{f}_{1}(x) \ldots \hat{f}_{n}(x)\right]^{T}$ such that $\|f(x)-\hat{f}(x)\|$ is small. Since $\|f(x)-\hat{f}(x)\|$ is small iff each of its components (which are nonlinear functions) is small, then by applying Theorem 3 proved in the previous section, the following corollary is obtained.

Theorem 4. For any smooth nonlinear system (11) satisfying the assumptions $S_{1}$ to $S_{2}$, it can be approximated, to any degree of accuracy, by a TSFMVC (10).

Similarly, smooth nonlinear control system

$$
\dot{x}=f(x)+g(x) u
$$

can also be approximated using a T-S fuzzy model with variable coefficient (TSFMVC) and is given by

$$
\dot{x}=\sum_{j_{1} j_{2} j_{3} \ldots j_{n}}^{r} h_{j_{1} j_{2} j_{3} \ldots j_{n}}(x)\left(A_{j_{1} j_{2} j_{3} \ldots j_{n}}(x) x+B_{j_{1} j_{2} j_{3} \ldots j_{n}}(x) u\right),
$$

where the rules are of the form:

$$
\begin{aligned}
R_{j_{1} j_{2} \ldots j_{n}}: \quad \text { IF } x_{1} \text { is about } j_{1} \epsilon \ldots \text { and } x_{n} \text { is about } j_{n} \epsilon \\
\text { THEN } \dot{x}=A_{j_{1} j_{2} \ldots j_{n}}(x) x+B_{j_{1} j_{2} \ldots j_{n}}(x) u,
\end{aligned}
$$

where $x=\left[x_{1}, x_{2}, \ldots, x_{n}\right]^{T}$ are the system states and $u=\left[u_{1}, u_{2}, \ldots, u_{n}\right]^{T}$ are the system inputs. $\dot{x}=A_{j_{1} j_{2} \ldots j_{n}}(x) x+B_{j_{1} j_{2} \ldots j_{n}}(x) u$ is the consequences of the $j_{1} j_{2} \ldots j_{n}{ }^{\text {th }}$ IF-THEN rule. The possibility for the $j_{1} j_{2} \ldots j_{n}^{\text {th }}$ rule to fire is given by $h_{j_{1} j_{2} j_{3} \ldots j_{n}}(x)=$ $\prod_{p=1}^{n} M_{j_{p}}\left(x_{p}\right)$.

\subsection{Comparison of Approximations Done in TSFMFC and TSFMVC}

Consider, for example, the function

$$
f\left(x_{1}, x_{2}\right)=\frac{\sin x_{1}}{1+\cos x_{1}}+\frac{x_{2}}{1+\sin x_{1}}
$$

where $0 \leqslant x_{1}, x_{2} \leqslant \pi / 4$.

The function approximated using the Takagi-Sugeno fuzzy model with fixed coefficient (TSFMFC) [also called as Takagi-Sugeno fuzzy model with linear coefficient (Takagi and Sugeno, 1985)] using two rules approximated at $x_{1}=0$ and $x_{1}=\pi / 4$ is as 
follows:

$$
\begin{aligned}
& \text { if } x_{1}=0, \text { then } f\left(x_{1}, x_{2}\right)=\left.A\right|_{x_{1}=0}\left[\begin{array}{l}
x_{1} \\
x_{2}
\end{array}\right] \\
& \text { if } x_{1}=\pi / 4, \text { then } f\left(x_{1}, x_{2}\right)=\left.A\right|_{x_{1}=\pi / 4}\left[\begin{array}{l}
x_{1} \\
x_{2}
\end{array}\right]
\end{aligned}
$$

where $\left.A\right|_{x_{1}=0}$ and $\left.A\right|_{x_{1}=\pi / 4}$ are coefficients of the function approximated at $x_{1}=0$ and $x_{1}=\pi / 4$ respectively and is given by

$$
\begin{aligned}
&\left.A\right|_{x_{1}=0}= {\left[\begin{array}{ll}
\frac{1}{2} & 1
\end{array}\right] \operatorname{since} \sin \left(x_{1}\right) \approx x_{1} \text { when } x_{1} \rightarrow 0 } \\
&\left.A\right|_{x_{1}=\pi / 4}=\left[\frac{4}{\pi(1+\sqrt{2})} \frac{\sqrt{2}}{1+\sqrt{2}}\right] \\
& \text { since } \sin \left(x_{1}\right) \approx \frac{4 x_{1}}{\pi \sqrt{2}} \text { when } x_{1} \text { is about } \pi / 4
\end{aligned}
$$

At the same time the function approximated using the proposed Takagi-Sugeno fuzzy model with variable coefficient (TSFMVC) using two rules approximated at $x_{1}=0$ and $x_{1}=\pi / 4$ is as follows:

$$
\begin{aligned}
& \text { if } x_{1}=0, \text { then } f\left(x_{1}, x_{2}\right)=\left.A(x)\right|_{x_{1}=0}\left[\begin{array}{l}
x_{1} \\
x_{2}
\end{array}\right] \\
& \text { if } x_{1}=\pi / 4, \text { then } f\left(x_{1}, x_{2}\right)=\left.A(x)\right|_{x_{1}=\pi / 4}\left[\begin{array}{l}
x_{1} \\
x_{2}
\end{array}\right],
\end{aligned}
$$

where $\left.A(x)\right|_{x_{1}=0}$ and $\left.A(x)\right|_{x_{1}=\pi / 4}$ are coefficients of the function approximated at $x_{1}=$ 0 and $x_{1}=\pi / 4$ respectively and is given by

$$
\begin{gathered}
\left.A(x)\right|_{x_{1}=0}=\left[\frac{1}{1+\cos x_{1}} \frac{1}{1+\sin x_{1}}\right] \text { since } \sin \left(x_{1}\right) \approx x_{1} \text { when } x_{1} \rightarrow 0 \\
\left.A(x)\right|_{x_{1}=\pi / 4}=\left[\frac{4 /(\pi \sqrt{2})}{1+\cos x_{1}} \frac{1}{1+\sin x_{1}}\right] \\
\text { since } \sin \left(x_{1}\right) \approx \frac{4 x_{1}}{\pi \sqrt{2}} \text { when } x_{1} \text { is about } \pi / 4
\end{gathered}
$$

It can be seen that (14) is again an approximation of (15) at $x_{1}$, i.e.,

$$
\begin{aligned}
& \left.A(x)\right|_{x_{1}=0} \text { at }\left(x_{1}=0\right)=\left.A\left(x \mid x_{1}=0\right)\right|_{x_{1}=0}=\left.A\right|_{x_{1}=0}, \\
& \left.A(x)\right|_{x_{1}=\pi / 4} \text { at }\left(x_{1}=\pi / 4\right)=\left.A\left(x \mid x_{1}=\pi / 4\right)\right|_{x_{1}=\pi / 4}=\left.A\right|_{x_{1}=\pi / 4} .
\end{aligned}
$$

In general, consider $j_{1} j_{2} \ldots j_{n}^{t h}$ rule of TSFMVC given by (3) and $j_{1} j_{2} \ldots j_{n}^{\text {th }}$ rule of TSFMVC given by (9). Then the approximation of $f(x)$ using fuzzy models 
TSFMVC (9) and TSFMFC (3) is as follows:

$$
\begin{aligned}
& f(x) \stackrel{\substack{1 \text { st } \\
\text { approx }}}{\longrightarrow} \sum_{j_{1} j_{2} \ldots j_{n}} h_{j_{1} j_{2} \ldots j_{n}}(x) a_{j_{1} j_{2} \ldots j_{n}}(x) x \\
& \stackrel{\text { 2nd }}{\text { approx. }} \longrightarrow\left\{\begin{array}{l}
\sum_{j_{1} j_{2} \ldots j_{n}} h_{j_{1} j_{2} \ldots j_{n}}(x) a_{j_{1} j_{2} \ldots j_{n}}\left(x_{j_{1} j_{2} \ldots j_{n}}\right) x \\
\text { or } \\
\sum_{j_{1} j_{2} \ldots j_{n}} h_{j_{1} j_{2} \ldots j_{n}}(x) a_{j_{1} j_{2} \ldots j_{n}} x .
\end{array}\right.
\end{aligned}
$$

It can be seen that the first approximation gives our proposed Takagi-Sugeno Fuzzy Model with Variable Coefficient (TSFMVC) and the second approximation leads to Takagi-Sugeno Fuzzy Model with Fixed Coefficient (TSFMFC, also called Takagi Sugeno Fuzzy Model with linear consequence).

Now consider the approximation of dynamic system of the form $\dot{x}=A(x)+B(x) u$ using fuzzy models.

$$
\begin{aligned}
\dot{x}= & A(x)+B(x) u \\
\stackrel{\substack{1 s t \\
\text { approx. }}}{\longrightarrow} \dot{x} & =\sum_{j_{1} j_{2} \ldots j_{n}} h_{j_{1} j_{2} \ldots j_{n}}(x)\left(A_{j_{1} j_{2} \ldots j_{n}}(x) x+B_{j_{1} j_{2} \ldots j_{n}}(x) u\right) \\
\stackrel{\substack{\text { 2nd } \\
\text { approx }}}{\longrightarrow} \dot{x} & =\left\{\begin{array}{l}
\sum_{j_{1} j_{2} \ldots j_{n}} h_{j_{1} j_{2} \ldots j_{n}}(x)\left(A_{j_{1} j_{2} \ldots j_{n}}\left(x_{j_{1} j_{2} \ldots j_{n}}\right) x+B_{j_{1} j_{2} \ldots j_{n}}\left(x_{j_{1} j_{2} \ldots j_{n}}\right) u\right) \\
\text { or } \\
\sum_{j_{1} j_{2} \ldots j_{n}} h_{j_{1} j_{2} \ldots j_{n}}(x)\left(A_{j_{1} j_{2} \ldots j_{n}} x+B_{j_{1} j_{2} \ldots j_{n}} u\right) .
\end{array}\right.
\end{aligned}
$$

In this case also it can be seen that the first approximation leads to our proposed TSFMVC. It is to be noted that TSFMFC is obtained only after the second approximation.

Thus TSFMFC are special cases of TSFMVC. Since the approximation error between the original system and the approximated system increases by the increase in the number of approximation, TSFMVC can better approximate systems than TSFMFC.

\section{Variable Gain Takagi-Sugeno Fuzzy Controller (VGTSFC)}

Parallel distributed compensation (Wang et al., 1995) can be used to design controller for Takagi-Sugeno fuzzy model with variable coefficient (TSFMVC) (13) and the variable gain PDC controller thus obtained is named as Variable Gain Takagi-Sugeno Fuzzy Controller (VGTSFC).

The rules of the VGTSFC are of the form:

$$
\begin{array}{ll}
\text { Rule } j_{1} j_{2} \ldots j_{n}: & \text { IF } x_{1} \text { is about } j_{1} \epsilon \ldots \text { and } x_{n} \text { is about } j_{n} \epsilon \\
& \text { THEN } u=K_{j_{1} j_{2} \ldots j_{n}}(x) x .
\end{array}
$$

The output of the controller is

$$
u=\sum_{j_{1} j_{2} \ldots j_{n}} h_{j_{1} j_{2} \ldots j_{n}}(x) K_{j_{1} j_{2} \ldots j_{n}}(x) x .
$$




\subsection{Comparison of Algorithms for FGTSFC and VGTSFC}

VGTSFC is designed based on TSFMVC ( $1^{\text {st }}$ approximated model), while FGTSFC is based on FGTSFC $\left(2^{\text {nd }}\right.$ approximated model). Since VGTSFC is designed based on the $1^{\text {st }}$ approximated model (TSFMVC) it can better control any system than FGTSFC. The comparison of algorithms for FGTSFC and VGTSFC are given in Table 1. It can be seen from the table that in FGTSFC the gain values $\left(K_{i} \mathrm{~s}\right)$ are fixed constants for each region $i$ and all the $K_{i}$ s are determined before the simulation. While in VGTSFC, the gain values are not fixed and it changes inside each region based on the current state of the system (or plant) and hence $K_{i}$ s are determined only during simulation.

\section{Translational Oscillation by a Rotational Actuator (TORA) - an Application}

TORA (translational oscillation by a rotational actuator) (Alleyne, 1998; Jankovic et al., 1996; Li et al., 2000; Kanellakopoulos and Zhao, 1995; Margaliot and Langholz, 2004; Rand et al., 1992) is a nonlinear benchmark problem corresponding to the physical system that has been built by Dr. Bernstein and his co-workers (Bernstein, 1998; Bupp et al., 1995) at the University of Michigan. It is illustrated in Fig. 1. The problem considers a translational oscillator with an attached eccentric rotational proof mass actuator, where the nonlinear coupling between the rotational motion of the actuator and the translational motion of the oscillator provides the control mechanism. The behavior TORA is similar to that of a dual-spin spacecraft exhibiting the resonance phenomenon.

Table 1

Algorithm for FGTSFC and VGTSFC

\begin{tabular}{|c|c|}
\hline Algorithm for FGTSFC & Algorithm for VGTSFC \\
\hline$\vdots$ & $\vdots$ \\
\hline$K_{i}=\operatorname{lqr}\left(A_{i}, B_{i}, Q, R\right), \forall i$ & begin \\
\hline begin & $t=1$ \\
\hline$t=1$ & $x_{t}=$ current state \\
\hline$x_{t}=$ current state & repeat \\
\hline repeat & : \\
\hline $\begin{array}{l}\vdots \\
u=-\sum_{i=1}^{r} h_{i}\left(x_{t}\right) K_{i} x_{t} \\
x_{t+1}=\operatorname{plant}\left(x_{t}, u\right)\end{array}$ & $\begin{array}{l}K_{i}=\operatorname{lqr}\left(A_{i}\left(x_{t}\right), B_{i}\left(x_{t}\right), Q, R\right), \forall i \\
u=-\sum_{i=1}^{r} h_{i}\left(x_{t}\right) K_{i} x_{t} \\
x_{t+1}=\operatorname{plant}\left(x_{t}, u\right)\end{array}$ \\
\hline 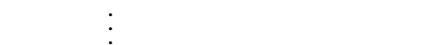 & 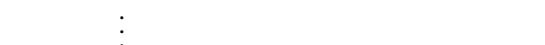 \\
\hline$t=t+i n c r$ & $t=t+i n c r$ \\
\hline until $t=$ maxtime & until $t=$ maxtime \\
\hline end & end \\
\hline
\end{tabular}




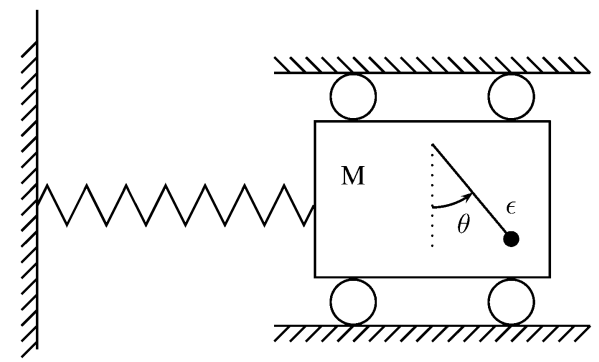

Fig. 1. Nonlinear benchmark problem - Translational Oscillation by a Rotational Actuator (TORA).

Let $x_{1}$ be the normalized displacement of the platform from the equilibrium position, $x_{2}=\dot{x_{1}}, x_{3}=\theta$ be the angle of the rotor, and $x_{4}=\dot{x_{3}}$. Then, the system dynamics can be expressed by the following equation:

$$
\dot{x}=f(x)+g(x) u,
$$

where $u$ is the torque applied to the eccentric mass and

$$
f(x)=\left[\begin{array}{c}
x_{2} \\
\frac{-x_{1}+\epsilon x_{4}^{2} \sin \left(x_{3}\right)}{1-\epsilon^{2} \cos ^{2}\left(x_{3}\right)} \\
x_{4} \\
\frac{\epsilon \cos \left(x_{3}\right)\left(x_{1}-\epsilon x_{4}^{2} \sin \left(x_{3}\right)\right.}{1-\epsilon^{2} \cos ^{2}\left(x_{3}\right)}
\end{array}\right], \quad g(x)=\left[\begin{array}{c}
0 \\
\frac{-\epsilon \cos \left(x_{3}\right)}{1-\epsilon^{2} \cos ^{2}\left(x_{3}\right)} \\
0 \\
\frac{1}{1-\epsilon^{2} \cos ^{2}\left(x_{3}\right)}
\end{array}\right] \text {, }
$$

where $\epsilon=0.5 m,-\pi / 2 \leqslant x_{3} \leqslant \pi / 2$ and $-1 \leqslant x_{4} \leqslant 1$. The equilibrium point of this system could be any point $\left[0,0, x_{3}, 0\right]$ among which only the point $[0,0,0,0]$ is the desired equilibrium point. The linearization around the point $[0,0,0,0]$ has two eigenvalues $\pm i$, which means that the TORA system is a critical nonlinear system.

In the following subsections, linear modeling and its controller, TSFMFC and FGTSFC, TSFMVC and VGTSFC and their simulations are presented.

\subsection{Linear Modeling and its Linear Controller}

The system is linearized around $x_{3}=0$ and $x_{4}=0$ is given by

$$
R_{1}:\left[\begin{array}{c}
\dot{x_{1}} \\
\dot{x_{2}} \\
\dot{x_{3}} \\
\dot{x_{4}}
\end{array}\right]=\left[\begin{array}{cccc}
0 & 1 & 0 & 0 \\
\frac{-1}{1-\epsilon^{2}} & 0 & 0 & 0 \\
0 & 0 & 0 & 1 \\
\frac{\epsilon}{1-\epsilon^{2}} & 0 & 0 & 0
\end{array}\right]\left[\begin{array}{l}
x_{1} \\
x_{2} \\
x_{3} \\
x_{4}
\end{array}\right]+\left[\begin{array}{c}
0 \\
\frac{-\epsilon}{1-\epsilon^{2}} \\
0 \\
\frac{1}{1-\epsilon^{2}}
\end{array}\right] u .
$$

Linear controller given by

$$
u=K x \text {. }
$$


Linear quadratic regulator method is used to find out the value $K$. The simulation and results are given in Subsection 6.4.

\subsection{T-S Modeling with Fixed Coefficient and its Fixed Gain Controller}

The system is approximated by the following 4-rule Takagi Sugeno fuzzy model with fixed coefficient (TSFMFC).

$R_{1}$ : If $x_{3}$ is about 0 and $x_{4}$ is about 0 , then

$$
\left[\begin{array}{l}
\dot{x_{1}} \\
\dot{x_{2}} \\
\dot{x_{3}} \\
\dot{x_{4}}
\end{array}\right]=\left[\begin{array}{cccc}
0 & 1 & 0 & 0 \\
\frac{-1}{1-\epsilon^{2}} & 0 & \frac{(0.01)^{2} \epsilon}{1-\epsilon^{2}} & 0 \\
0 & 0 & 0 & 1 \\
\frac{\epsilon}{1-\epsilon^{2}} & 0 & \frac{-(0.01)^{2} \epsilon^{2}}{1-\epsilon^{2}} & 0
\end{array}\right]\left[\begin{array}{l}
x_{1} \\
x_{2} \\
x_{3} \\
x_{4}
\end{array}\right]+\left[\begin{array}{c}
0 \\
\frac{-\epsilon}{1-\epsilon^{2}} \\
0 \\
\frac{1}{1-\epsilon^{2}}
\end{array}\right] u
$$

$R_{2}$ : if $x_{3}$ is about 0 and $x_{4}$ is about \pm 1 , then

$$
\left[\begin{array}{c}
\dot{x_{1}} \\
\dot{x_{2}} \\
\dot{x_{3}} \\
\dot{x_{4}}
\end{array}\right]=\left[\begin{array}{cccc}
0 & 1 & 0 & 0 \\
\frac{-1}{1-\epsilon^{2}} & 0 & \frac{\epsilon}{1-\epsilon^{2}} & 0 \\
0 & 0 & 0 & 1 \\
\frac{\epsilon}{1-\epsilon^{2}} & 0 & \frac{-\epsilon^{2}}{1-\epsilon^{2}} & 0
\end{array}\right]\left[\begin{array}{l}
x_{1} \\
x_{2} \\
x_{3} \\
x_{4}
\end{array}\right]+\left[\begin{array}{c}
0 \\
\frac{-\epsilon}{1-\epsilon^{2}} \\
0 \\
\frac{1}{1-\epsilon^{2}}
\end{array}\right] u,
$$

$R_{3}$ : if $x_{3}$ is about $\pm \pi / 2$ and $x_{4}$ is about 0 , then

$$
\left[\begin{array}{c}
\dot{x_{1}} \\
\dot{x_{2}} \\
\dot{x_{3}} \\
\dot{x_{4}}
\end{array}\right]=\left[\begin{array}{cccc}
0 & 1 & 0 & 0 \\
\frac{-1}{1-\epsilon^{2} \beta^{2}} & 0 & \frac{2(0.01)^{2} \epsilon}{\pi\left(1-\epsilon^{2} \beta^{2}\right)} & 0 \\
0 & 0 & 0 & 1 \\
\frac{\epsilon \beta}{1-\epsilon^{2} \beta^{2}} & 0 & \frac{-2(0.01)^{2} \epsilon^{2} \beta}{\pi\left(1-\epsilon^{2} \beta^{2}\right)} & 0
\end{array}\right]\left[\begin{array}{l}
x_{1} \\
x_{2} \\
x_{3} \\
x_{4}
\end{array}\right]+\left[\begin{array}{c}
0 \\
\frac{-\epsilon \beta}{1-\epsilon^{2} \beta^{2}} \\
0 \\
\frac{1}{1-\epsilon^{2} \beta^{2}}
\end{array}\right] u
$$

$R_{4}$ : if $x_{3}$ is about $\pm \pi / 2$ and $x_{4}$ is about \pm 1 , then

$$
\left[\begin{array}{c}
\dot{x_{1}} \\
\dot{x_{2}} \\
\dot{x_{3}} \\
\dot{x_{4}}
\end{array}\right]=\left[\begin{array}{cccc}
0 & 1 & 0 & 0 \\
\frac{-1}{1-\epsilon^{2} \beta^{2}} & 0 & \frac{2 \epsilon}{\pi\left(1-\epsilon^{2} \beta^{2}\right)} & 0 \\
0 & 0 & 0 & 1 \\
\frac{\epsilon \beta}{1-\epsilon^{2} \beta^{2}} & 0 & \frac{-2 \epsilon^{2} \beta}{\pi\left(1-\epsilon^{2} \beta^{2}\right)} & 0
\end{array}\right]\left[\begin{array}{l}
x_{1} \\
x_{2} \\
x_{3} \\
x_{4}
\end{array}\right]+\left[\begin{array}{c}
0 \\
\frac{-\epsilon \beta}{1-\epsilon^{2} \beta^{2}} \\
0 \\
\frac{1}{1-\epsilon^{2} \beta^{2}}
\end{array}\right] u
$$

where $\beta=\cos \left(88^{\circ}\right)$.

Note: Note that each rule consequent is of the form $\dot{x}=A_{i} x+B_{i} u$.

By utilizing the concept of Parallel Distributed Compensation (PDC), the following four rules are designed for the FGTSFC.

$R_{1}$ : if $x_{3}$ is about 0 and $x_{4}$ is about 0 , then $u_{1}=K_{1} x$,

$R_{2}$ : if $x_{3}$ is about 0 and $x_{4}$ is about \pm 1 then $u_{2}=K_{2} x$,

$R_{3}$ : If $x_{3}$ is about $\pm \pi / 2$ and $x_{4}$ is about 0 , then $u_{3}=K_{3} x$,

$R_{4}$ : if $x_{3}$ is about $\pm \pi / 2$ and $x_{4}$ is about \pm 1 , then $u_{4}=K_{4} x$.

Linear quadratic regulator method is used to find out the values of $K_{i}^{\prime}$ s. The simulation and results are given in Subsection 6.4. 


\subsection{T-S Modeling with Variable Coefficient and its Variable Gain Controller}

In this subsection, VGTSFC with 2 rule model is presented. In two rule model, two of the state variables in the coefficient matrix are kept varying, i.e., $x_{3}$ and $x_{4}$.

$R_{1}$ : if $x_{3}$ is about 0 , then

$$
\left[\begin{array}{c}
\dot{x_{1}} \\
\dot{x_{2}} \\
\dot{x_{3}} \\
\dot{x_{4}}
\end{array}\right]=\left[\begin{array}{cccc}
0 & 1 & 0 & 0 \\
\frac{-1}{1-\epsilon^{2} \cos ^{2}\left(x_{3}\right)} & 0 & \frac{x_{4}^{2} \epsilon}{1-\epsilon^{2} \cos ^{2}\left(x_{3}\right)} & 0 \\
0 & 0 & 0 & 1 \\
\frac{\epsilon \cos \left(x_{3}\right)}{1-\epsilon^{2} \cos ^{2}\left(x_{3}\right)} & 0 & \frac{-x_{4}^{2} \epsilon^{2} \cos \left(x_{3}\right)}{1-\epsilon^{2} \cos ^{2}\left(x_{3}\right)} & 0
\end{array}\right]\left[\begin{array}{c}
x_{1} \\
x_{2} \\
x_{3} \\
x_{4}
\end{array}\right]+\left[\begin{array}{c}
0 \\
\frac{-\epsilon \cos \left(x_{3}\right)}{1-\epsilon^{2} \cos ^{2}\left(x_{3}\right)} \\
0 \\
\frac{1}{1-\epsilon^{2} \cos ^{2}\left(x_{3}\right)}
\end{array}\right] u,
$$

$R_{2}$ : if $x_{3}$ is about $\pm \pi / 2$, then

$$
\left[\begin{array}{c}
\dot{x_{1}} \\
\dot{x_{2}} \\
\dot{x_{3}} \\
\dot{x_{4}}
\end{array}\right]=\left[\begin{array}{cccc}
0 & 1 & 0 & 0 \\
\frac{-1}{1-\epsilon^{2} \cos ^{2}\left(x_{3}\right)} & 0 & \frac{2 x_{4}^{2} \epsilon}{\pi\left(1-\epsilon^{2} \cos ^{2}\left(x_{3}\right)\right)} & 0 \\
0 & 0 & 0 & 1 \\
\frac{\epsilon \cos \left(x_{3}\right)}{1-\epsilon^{2} \cos ^{2}\left(x_{3}\right)} & 0 & \frac{-2 x_{4}^{2} \epsilon^{2} \cos \left(x_{3}\right)}{\pi\left(1-\epsilon^{2} \cos ^{2}\left(x_{3}\right)\right)} & 0
\end{array}\right]\left[\begin{array}{l}
x_{1} \\
x_{2} \\
x_{3} \\
x_{4}
\end{array}\right]+\left[\begin{array}{c}
0 \\
\frac{-\epsilon \cos \left(x_{3}\right)}{1-\epsilon^{2} \cos ^{2}\left(x_{3}\right)} \\
0 \\
\frac{1}{1-\epsilon^{2} \cos ^{2}\left(x_{3}\right)}
\end{array}\right] u \text {. }
$$

By utilizing the concept of Parallel Distributed Compensation (PDC), the following two rules are designed for the variable gain T-S fuzzy controller.

$$
\begin{aligned}
& R_{1}: \text { if } x_{3} \text { is about } 0, \text { then } u_{1}=K_{1}(x) x, \\
& R_{2}: \text { if } x_{3} \text { is about } \pm \pi / 2 \text {, then } u_{2}=K_{2}(x) x .
\end{aligned}
$$

Linear quadratic regulator method is used to find out the values of $K_{i}(x)^{\prime} s$. The simulation and results are given in Subsection 6.4.

\subsection{Simulation and Results}

This subsection presents the simulation results and discussions of linear controller (Subsection 6.1), FGTSFC (Subsection 6.2), and VGTSFC (Subsection 6.3). Linear quadratic regulator method is used to find out the values $K_{i}$ of above controllers.

$$
\text { Let } Q=\left[\begin{array}{cccc}
10 & 0 & 0 & 0 \\
0 & 0 & 0 & 0 \\
0 & 0 & 1 & 0 \\
0 & 0 & 0 & 0
\end{array}\right] \text { and } R=1 \text {. }
$$

For the above values of $\mathrm{Q}$ and $\mathrm{R}$, the linear quadratic method gives the the following value for the gain $\mathrm{K}$ of the linear controller described in Subsection 6.1

$$
K=\left[\begin{array}{llll}
-1.7510 & 2.2766 & 2.7463 & 3.4691
\end{array}\right]
$$

The gain values of FGTSFC (described in Subsection 6.2) are obtained as

$$
K_{1}=\left[\begin{array}{llll}
-2.6490 & 0.5780 & 1.0001 & 2.1563
\end{array}\right]
$$




$$
\begin{aligned}
K_{2} & =\left[\begin{array}{llll}
-1.7510 & 2.2766 & 2.7463 & 3.4691
\end{array}\right] \\
K_{3} & =\left[\begin{array}{llll}
-3.1448 & 0.0129 & 1.0001 & 1.4526
\end{array}\right] \\
K_{4} & =\left[\begin{array}{llll}
-2.6071 & 1.7641 & 2.2487 & 2.1725
\end{array}\right]
\end{aligned}
$$

The gain values of VGTSFC (described in Subsection 6.3) is dynamic, not unique and it changes with the state of the system and have to be calculated at runtime.

The simulation of TORA system is carried out on Celeron $2 \mathrm{GHz}$ machine using MATLAB 6. To simulate the TORA dynamics, a fourth-order Runge-Kutta method (ode45 (Shampine and Reichelt, 1997)) is used with an integration time step size of $0.01 \mathrm{sec}$. Observation of the system states were made every time step (integration step). The controllers are assumed to be continuous, therefore, the sampling time of the controller was set equal to the integration time step size.

TORA system is simulated with linear controller, FGTSFC and, VGTSFC for several initial conditions. Figs. 2, 3, 4, and 5 respectively show the plot of displacement $\left(x_{1}\right)$, Velocity $\left(x_{2}\right)$ vs Displacement $\left(x_{1}\right)$, angle $\left(x_{3}\right)$ vs angular velocity $\left(x_{4}\right)$, and control force for the initial state $\left[0,0,85^{\circ}, 0\right]$ with each of the above mentioned controllers. Table 2 shows the performance of the Linear controller, FGTSFC and, VGTSFC for the initial state $\left[0,0,85^{\circ}, 0\right]$ where the Total Absolute Error (TAE) and Performance index (J) is calculated by (24) and (25).

$$
T A E=\sum_{t=0: 0.01: \text { maxtime }}\left(\left|x_{1}(t)\right|+\left|x_{2}(t)\right|+\left|x_{3}(t)\right|+\left|x_{4}(t)\right|\right),
$$

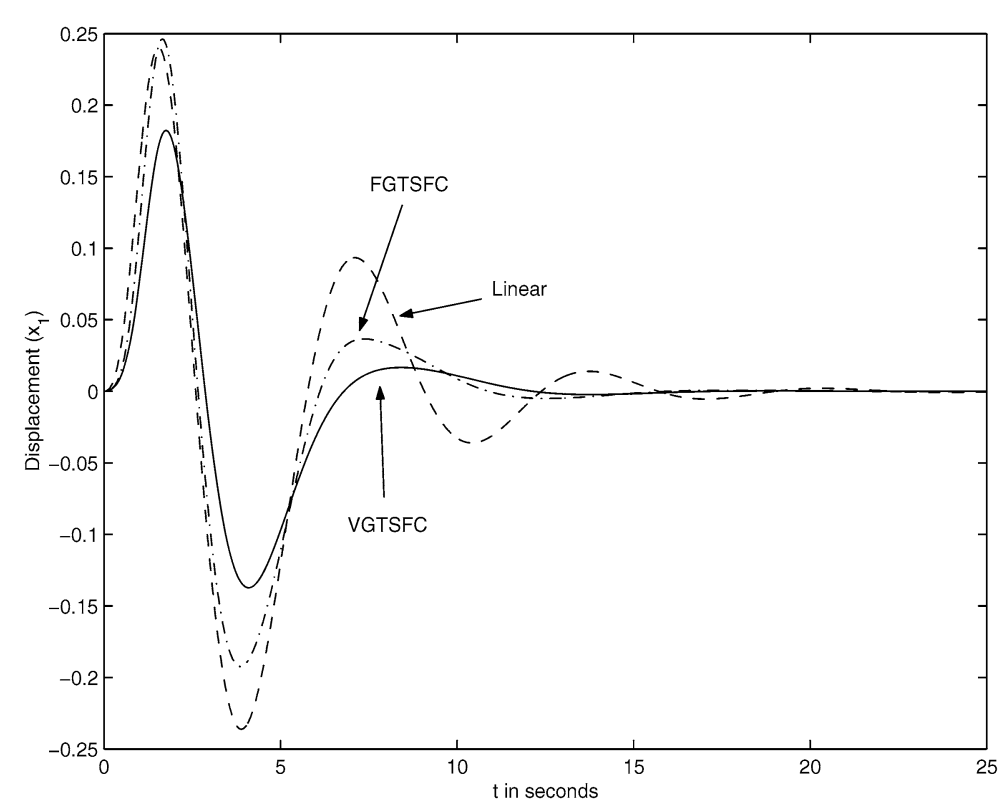

Fig. 2. Displacement ( $x_{1}$ in meters) of TORA system for the initial state $\left[0,0,85^{\circ}, 0\right]$. 


$$
J=\sum_{t=0: 0.01: \text { maxtime }}\left(x^{T} Q x+u^{T} R u\right) .
$$

It is clear from the Fig. 2 that VGTSFC has lesser displacement compared to Linear controller and FGTSFC. From Fig. 3, it is clear that the area taken by VGTSFC is much lesser when compared to Linear controller and FGTSFC. From Fig. 4, it is clear that the variable $x_{3}$ and $x_{4}$ also performs well in the case of VGTSFC. It can also be seen from the Fig. 5 that the control force of the VGTSFC is much less when compared to other controllers. It is evident from the Table 2 that Variable Gain Takagi-Sugeno Fuzzy Controllers (VGTSFC) is having lesser error and hence perform much better than the linear controller and Fixed Gain Takagi-Sugeno Fuzzy Controller (FGTSFC).

Simulation results reveals that the Variable Gain Takagi-Sugeno Fuzzy Controller (VGTSFC) can efficiently be used in place of linear controllers and fixed gain TakagiSugeno fuzzy controllers with good approximation and control performance.

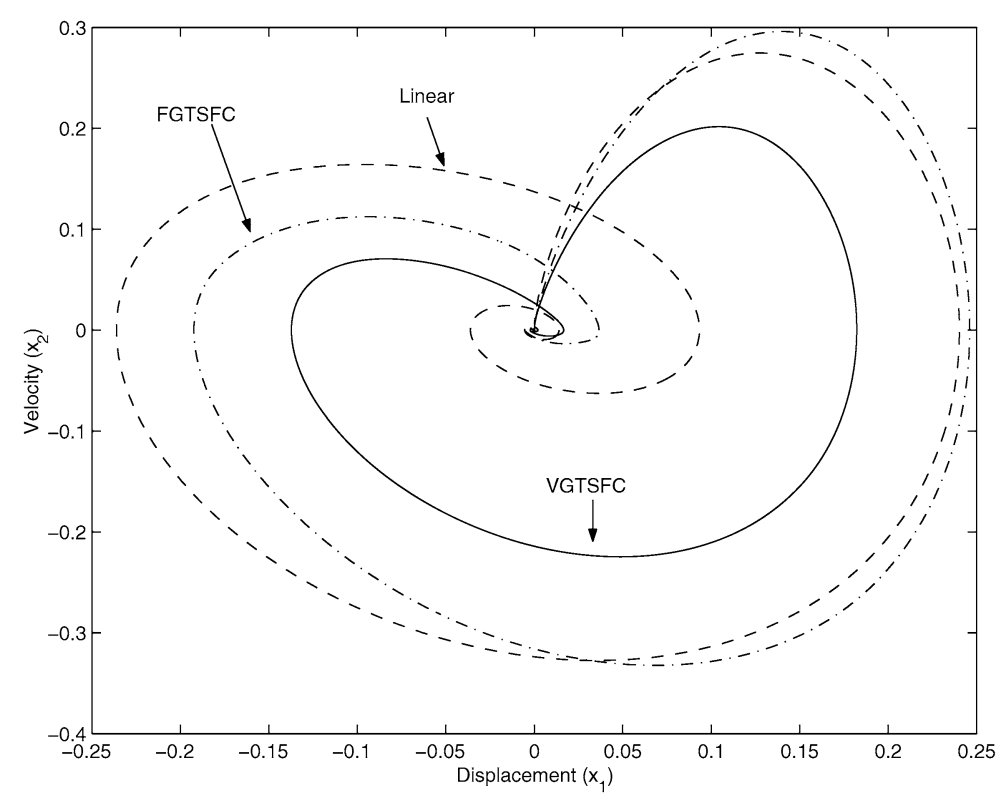

Fig. 3. Velocity $\left(x_{2}\right.$ in $\left.\mathrm{m} / \mathrm{s}\right)$ vs Displacement $\left(x_{1}\right.$ in $\left.\mathrm{m}\right)$ for the initial state $\left[0,0,85^{\circ}, 0\right]$.

Table 2

Performance of linear controller, FGTSFC and VGTSFC

\begin{tabular}{lcc}
\hline Methods & Total Absolute Error (TAE) & Performance Index (J) \\
\hline Linear & 702.9854 & 609.4228 \\
\hline FGTSFC & 692.3458 & 521.8640 \\
\hline VGTSFC & 648.5775 & 432.0092 \\
\hline
\end{tabular}




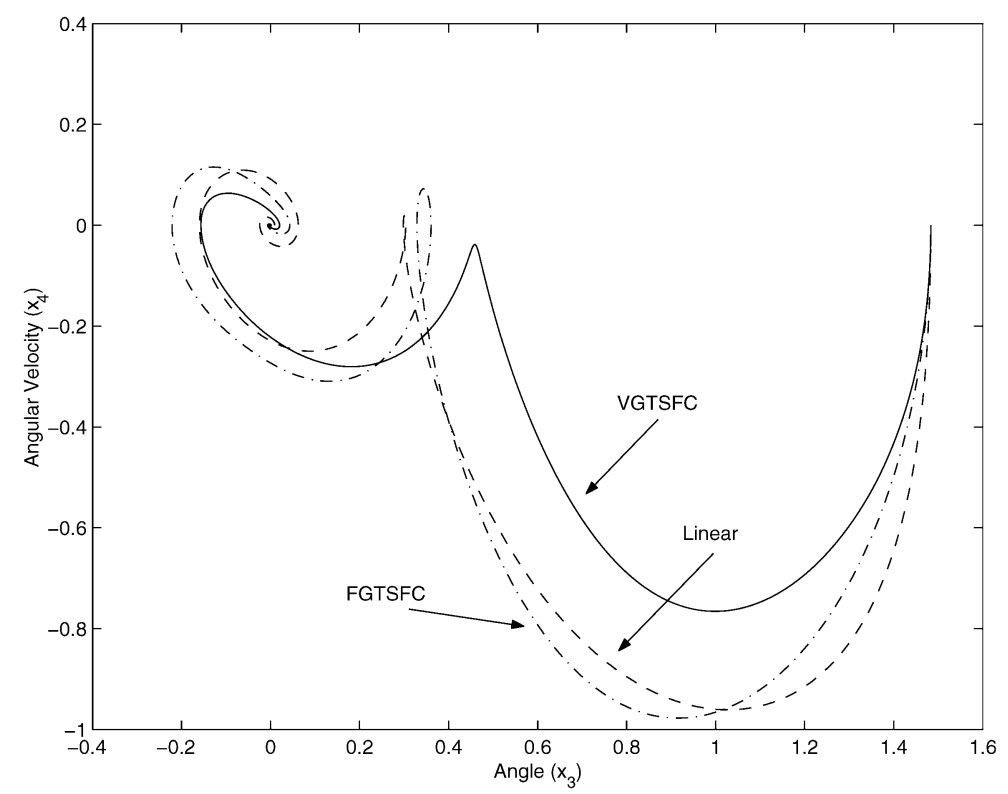

Fig. 4. Angle $\left(x_{3}\right)$ vs Angular velocity $\left(x_{4}\right)$ of TORA system for the initial state $\left[0,0,85^{\circ}, 0\right]$.

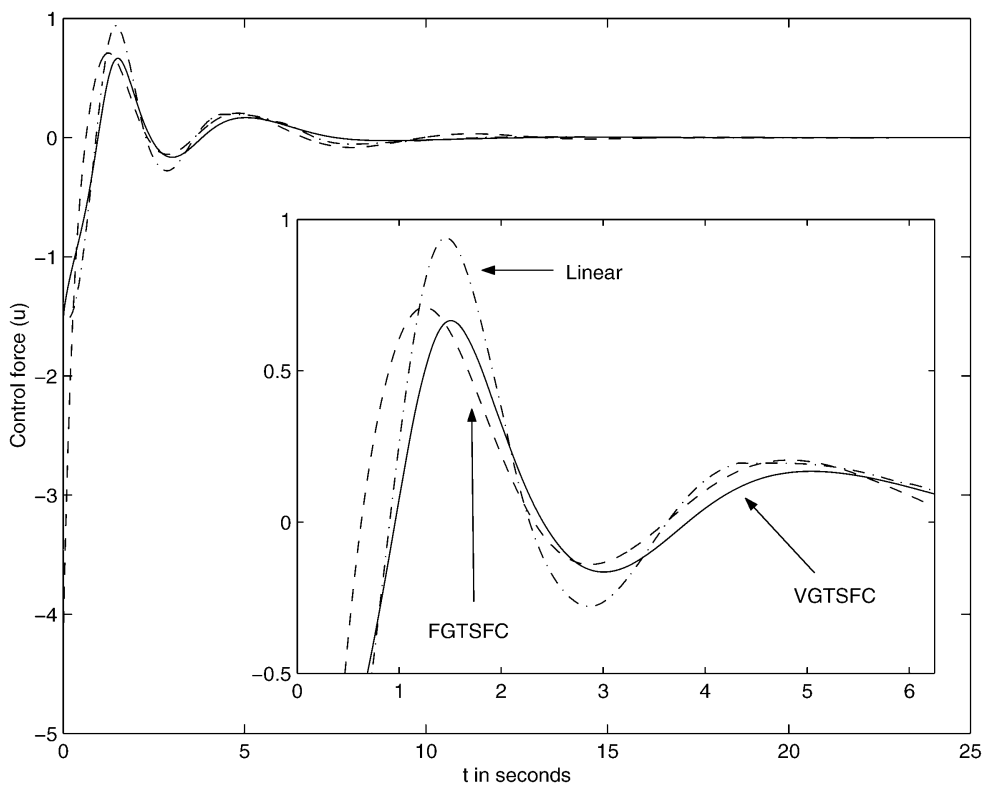

Fig. 5. Control force ( $u$ in Newton) applied to the TORA system for the initial state $\left[0,0,85^{\circ}, 0\right]$. A portion of the plot is zoomed and is shown inside the figure. 


\section{Conclusions}

A new model namely, Takagi-Sugeno Fuzzy Model with Variable Coefficient (TSFMVC) is presented and it is proved that it can approximate any nonlinear function to any degree of accuracy. A new controller namely, Variable Gain Takagi-Sugeno Fuzzy Controller (VGTSFC) is presented and compared with linear and fixed gain Takagi-Sugeno controllers. The simulation results of TORA system show that the proposed controller is effective and efficient.

The main advantages of the modeling (TSFMVC) and control (VGTSFC) are summarized below.

1. TSFMVC can better approximate a nonlinear dynamic system than TSFMFC and there by reducing the approximation error in modeling.

2. Variable gain Takagi-Sugeno fuzzy controller (VGTSFC), developed on the basis of the Takagi-Sugeno fuzzy modeling with variable coefficient (TSFMVC), shows good control performance when compared to linear controller and fixed gain Takagi-Sugeno fuzzy controllers (FGTSFC).

\section{References}

Alleyne, A. (1998). Physical insights on passivity-based TORA control designs. IEEE Trans. Control Syst. Technol., 6, 436-439.

Bernstein, D.S. (Ed.) (1998). Special issue: A nonlinear benchmark problem. Int. J. Robust Nonlinear Control, 8.

Bupp, R.T., D.S. Bernstein and V.T. Coppola (1995). A benchmark problem for nonlinear control design: problem statement, experiment testbed, and passive nonlinear compensation. In Proc. 1995 American Control Conference. pp. 4363-4368.

Jankovic, M., D. Fontaine and P.V. Kokotovic (1996). TORA example: Cascade and Passivity-based control designs. IEEE Trans. Control Syst. Technol, 4, 292-297.

Joh, J., Y.-H. Chen and R. Langari (1998). On the stability issues of linear Takagi-Sugeno fuzzy models. IEEE Trans. on Fuzzy systems, 6, 402-410.

Joh, J., and R. Langari (2000). A frame work for the synthesis of PDC - Type Takagi-Sugeno Fuzzy Control systems - An LMI approach. In S.S. Farinwata, D. Filev, R. Langari (Eds.), Fuzzy Control Synthesis and Analysis. Wiley.

Kanellakopoulos, I., and J.X. Zhao (1995). Tracking and distrubance rejection for the benchmark nonlinear control problem. In Proc. 1995 American Control Conference. pp. 4360-4362.

Li, J., H.O. Wang, L. Bushnell, Y. Hong and K. Tanaka (2000). A fuzzy logic approach to optimal control of nonlinear systems. Int. J. of Fuzzy Systems, 2(3), 153-163.

Li, N., S.Y. Li, Y.G. Xi, S.S. Ge (2003). Stability analysis of T-S fuzzy system based observers. Int. J. of Fuzzy Systems, 5(1), 22-30.

Margaliot, M., and G. Langholz (2004). Fuzzy control of a benchmark problem: a computing with words approach. IEEE Trans. Fuzzy Systems, 12(2), 230-235.

Rand, R.H., R.J. Kinsey and D.L. Mingori (1992). Dynamics of spinup through resonance. Int. J. Nonlinear Mech., 27(3), 489-502.

Shampine, L.F., and M.W. Reichelt (1997). The MATLAB ODE suite. SIAM Journal on Scientific Computing, 18(1).

Takagi, T., and M. Sugeno (1985). Fuzzy identification of systems and its applications to modeling and control. IEEE Trans. System, Man and Cybernetics, 15(1), 116-132.

Tanaka, K., and M. Sugeno (1992). Stability analysis and design of fuzzy control systems. Fuzzy Sets and Systems, 45(2), 135-156. 
Wang, H.O., K. Tanaka and M. Griffin (1995). Parallel distributed compensation of nonlinear systems by Takagi-Sugeno fuzzy model. IEEE, 531-538.

Wang, H.O., K. Tanaka and M.F. Griffin (1996). An approach to fuzzy control of nonlinear systems: stability and design issues. IEEE Trans. on Fuzzy Systems, 4(1), 14-23.

Wang, H.O., J. Li and K. Tanaka (2003). T-S fuzzy model with linear rule consequence and PDC sontroller: A universal framework for nonlinear control systems. International Journal of Fuzzy Systems, 5(2), 106-113.

Ying, H. (1998). Constructing nonlinear variable gain controllers via the Takagi-Sugeno fuzzy control. IEEE Trans. on Fuzzy Systems, 6(2), 226-234.

R. Rajesh was awarded the BSc degree in mathematics, MSc degree in computer science and $\mathrm{PhD}$ in computer science in 1996, 1998 and 2005 respectively from University of Kerala, India. He is currently working as a lecturer in the Department of Computer Science and Engineering of the Bharathiar University, India. He has for his credit more than 9 conference/journal papers. His research interests include the fuzzy logic, genetic algorithm, image processing, neural networks, robotics, natural language processing, etc.

M.R. Kaimal received $\mathrm{PhD}$ from Mehta Research Institute (University of Allahabad) in 1978. During 1977-79 he worked in Indian Institute of Science, Bangalore as a research associate. He was a visiting fellow at National Institutes of Health, Bethesda, Maryland (1981-84) on John Fogarty International fellowship and also worked in CUMC, Cornell (1984). He had been in the Faculty of Cochin University of Science and Technology, Kerala, till 1987. Since 1987 he is with the Department of Computer Science, University of Kerala, Thiruvananthapuram where currently he is a professor and head of the Department of Computer Science. He has for his credits more than 50 international journal/conference and national journal/conference papers. His research interests include the fuzzy logic, genetic algorithm, image processing, neural networks, robotics, natural language processing, signal processing, medical informatics, speech processing, biological modeling, data structures, etc.

\section{Kintamo naudingumo Takagi-Sugeno fuzzy logikos kontroleriai}

Reghunadhan RAJESH, M.Ramachandra KAIMAL

Daugelis literatūroje minimu Takagi-Sugeno Fuzzy (TSF) sistemų naudoja tik tiesines iejjimo kintamuju funkcijas. Jos gali būti vadinamos TSF modeliais su fiksuotais koeficientais (TSFMFC). Šiame straipsnyje pateiktas TSF modelis su kintamu koeficientu (TSFMVC), kuris gali artimiau aproksimuoti netiesinių sistemų, netiesinių dinaminių sistemų ir netiesinių valdymo sistemų klases. Taip pat parodyta, kad TSFMFC yra dalinis TSFMVC atvejis. Be to, yra aprašytas kintamo naudingumo kontroleris (VGTSFC), jis veikia geriau nei fiksuoto naudingumo TSF kontroleris (FGTSFC). 